Résumés des conférences et travaux

\title{
Histoire de la France féodale
}

\section{Dominique Barthélemy}

\section{OpenEdition \\ Journals}

\section{Édition électronique}

URL : https://journals.openedition.org/ashp/3872

DOI : $10.4000 /$ ashp.3872

ISSN : 1969-6310

\section{Éditeur}

Publications de l'École Pratique des Hautes Études

\section{Édition imprimée}

Date de publication : 1 septembre 2020

Pagination : 262-266

ISSN : 0766-0677

\section{Référence électronique}

Dominique Barthélemy, "Histoire de la France féodale ", Annuaire de l'École pratique des hautes études (EPHE), Section des sciences historiques et philologiques [En ligne], 151 | 2020, mis en ligne le 09 juillet 2020, consulté le 06 juillet 2021. URL : http://journals.openedition.org/ashp/3872 ; DOI : https:// doi.org/10.4000/ashp.3872 


\title{
HISTOIRE DE LA FRANCE FÉODALE
}

\author{
Directeur d'études : M. Dominique BarthéLEMY, \\ correspondant de l'Institut
}

Programme de l'année 2018-2019 : I. Échanges et confrontations avec la Lorraine et l'Allemagne. - II. Questions diverses.

La première conférence étudie d'abord quatre épisodes importants des relations entre le royaume capétien et l'empire : les interventions ottoniennes de 946-949 et de 978, relatées notamment par Richer de Reims (Histoires II.17-19, 29-31 et 53-54, III.67 à 78), l'entrevue de Mouzon et Ivois entre Henri II et Rolert le Pieux en 1123 (Raoul Glaber, Histoires, III 7-8) et la menace de l'empereur Henri V sur Reims, repoussée en 1124 par une mobilisation fameuse de Louis VI. Dans ce dernier cas, on relève tout de même qu'il s'agissait surtout de faire diversion en empêchant Louis VI d'entrer dans la Normandie d'Henri Beau Clerc, beau-père de l'empereur; en outre, l'avantage moral du roi de France paraît avoir été largement contrebalancé par sa dangereuse acceptation, à cette occasion, de la réunion de la Champagne au comté de Blois entre les mains de Thibaud IV le Grand.

D'autre part, on entend un exposé du docteur Jens Schneider sur le Ludwigslied célébrant la victoire de Louis III à Saucourt sur les Normands (882), en langue allemande aux fins de ralliement de la Francie orientale, et une intervention conjointe des professeurs René Pérennec et Jean-René Valette sur la métamorphose du Perceval de Chrétien de Troyes en un Parzival dû à Wolfram von Eschenbach.

Enfin le directeur d'études s'interroge sur l'acclimatation fugitive de la paix diocésaine et de la trêve de Dieu en terres d'empire. Y avait-il là, au $\mathrm{XI}^{\mathrm{e}}$ siècle, la même densité de guerres féodales? Notre documentation de l'an mil suggère du moins quelques contrastes, qui pourraient bien rendre compte du fait que le code des sauvegardes contre la guerre féodale (= paix diocésaine) ne s'est guère diffusé hors des Gaules, alors que la trêve de Dieu, plus soucieuse de l'homicide, avait davantage vocation à pénétrer dans l'empire.

Des serments de paix selon la formule fameuse de 1019 / 1023 ne semblent pas avoir été prêtés en Lorraine et Allemagne. Les historiens modernes ont jugé très significatif qu'un évêque impérial, Gérard de Cambrai, soulève des objections de principe avant de céder à la pression venue d'Arras et d'admettre le serment dans son diocèse à cheval sur la frontière avec la France capétienne. Pourtant ces objections, telles que les rapportent les Gesta, surprennent un peu : Gérard de Cambrai aurait dit craindre l'abus du serment et l'engagement des évêques dans une mission d'ordre public relevant du roi, seul, alors même que les évêques impériaux sont tout de même très engagés dans ce sens, et ne répugnent pas à demander des serments d'allégeance et d'amitié, souvent trahis (Gesta episcoporum cameracensium III.27 : MGH SS VII, p. 474). Il n'en est pas moins vrai que la «paix » des évêques de Bourgogne et de 
France aide à maîtriser des conflits plus circonscrits et des pillages probablement plus fréquents que ceux de la Lorraine et de l'Allemagne; et elle peut impliquer la mobilisation par l'évêque d'armées diocésaines plus larges que les contingents vassaliques, avec tous les risques politiques, sociaux et moraux que cela implique.

Observons toutefois que des évêques italiens, piémontais au moins, n'ont pas hésité à rassembler des reliques pour un concile de paix au moins, tenu en un lieu (Verraria ou Verrua) et à une date difficiles à préciser (celle de 1038 n'est pas assurée). Et comme toujours lorsque nous n'avons qu'un écho hagiographique, nous n'en connaissons pas les décrets. La trêve de Dieu a eu, quant à elle, un écho bref, mais indéniable, en Italie, par suite de la lettre adressée aux Italiens (sans doute sur leur demande) en 1037 / 1041 par l'archevêque Raimbaud d'Arles avec trois évêques et l'abbé Odilon de Cluny. En revanche, rien ne prouve son adoption en Allemagne avant 1082 .

Les historiens modernes ont bien relevé la coïncidence entre le premier essor de la trêve de Dieu aux années 1040, jusqu'en Lorraine, et une entreprise impériale de paix chrétienne sans précédent, mais s'agit-il de paix et trêve de Dieu, ou même d'une réplique à ces législations? Des annales et une lettre donnent le synode de Constance de 1043 pour le temps fort de cette entreprise. Alors que l'affrontement du Capétien Henri I ${ }^{\text {er }}$ avec les fils d'Eudes II de Blois a arrêté sans cérémonie la trêve de Dieu en France occidentale, ne peut-on créditer le Salien Henri III d'avoir endigué une possible diffusion de la " paix de Dieu » (paix et trêve amalgamées) dans son empire en s'engageant dans une entreprise rivale? À Constance et à Trêves en 1043, il pardonne solennellement à ses sujets leurs torts envers lui et il les engage à faire de même entre eux, par des « pactes de paix ».

Une paix inédite, celle-là même que des anges apportèrent du Ciel à la Nativité du Christ et que celui-ci laissa à ses disciples, cela consonne en effet avec la lettre aux Italiens, comme avec la paix d'entre 996 et 1014 du duc Guillaume V d'Aquitaine (dont Henri III épouse alors une fille). Une dynamique de réconciliations entre chrétiens, voilà qui est bien aussi dans le même esprit. Cependant la ressemblance ne frappe que si l'on croit a priori que les hommes de l'an mil et du $\mathrm{XI}^{\mathrm{e}}$ siècle avaient presque perdu toute notion, tout espoir de paix. Car pareilles évocations et pareille exigence morale sont très naturellement chrétiennes, et les écoles épiscopales ou monastiques de l'empire (comme celle de Reichenau) n'ont pas besoin qu'un vent de sud-ouest, providentiel, les leur apporte d'Aquitaine. Le souci pacificateur d'Henri III est au cœur de sa mission royale, et sa vigueur tient aussi à la difficulté d'une guerre contre des Hongrois, en des campagnes qui précèdent et suivent le synode de Constance et au cours desquelles l'unité de son armée a besoin d'être renforcée. Est-ce d'ailleurs si valorisant pour Henri III, que d'abandonner (provisoirement en fait) la posture impériale qui traiterait en rébellions les inimitiés contre lui et les sanctionnerait, même faiblement, au lieu de les pardonner? Est-ce d'un empereur, que de se ravaler ainsi au rang de ses sujets?

On cherche en vain une trace durable en Allemagne des pactes de paix de 10431044, qui ont amnistié des seigneurs sans les inviter à s'amender de leurs méfaits. Leur succès n'a rien d'évident, même à moyen terme. Dans la suite de son règne, Henri III est considéré comme un empereur de guerre et de paix, en Bourgogne, à 
l'instar de ses prédécesseurs. Quant à la culture politique au début du règne de son fils de Henri IV, telle du moins que la présente Lambert de Hersfeld, elle redevient franchement séculière, même si une réconciliation embellit à Goslar la fête de Noël 1068 (MGH SS V p. 429 et 274).

Si quelque chose, tout de même, dans le règne d'Henri III, se rapproche en partie d'un serment de paix à la manière des Gaules, je ne vois que le serment prêté pour dix ans, que Ludwig Weiland, dans son édition de 1893, appelle pax italica et attribue à Henri IV vers 1077, bien que le texte se réfère clairement au «troisième Henri » (MGH Constitutiones I, $\mathrm{n}^{\circ}$ 68). L'érudition récente, tant allemande qu'italienne, a rendu ce texte au règne d'Henri III; elle n'a cependant pas aperçu une certaine analogie, mutatis mutandis, avec les paix diocésaines du royaume capétien. En revanche, ce serment italien à Henri III ou ses délégués a beaucoup moins de relation à une trêve de Dieu, puisqu'il évoque des violences autres que l'homicide, à empêcher ou réprimer en tout temps.

La deuxième conférence se place dans le prolongement de l'étude, menée l'année dernière, de la charte toulousaine de 1163 retrouvée dans le fonds Giry de l'EPHE (Annuaire 2017-2018, p. 290-293), et s'attache à réexaminer d'autres dossiers de la paix et trêve de Dieu après 1130 en Occitanie.

Des statuts de paix conservés au cartulaire de Dax ont réapparu en 2004 (Archives du diocèse de Dax, sans cotation, f. 34-35v. Jean Cabanot et Georges Pon, éd. Cartulaire de la cathédrale de Dax. Liber Rubeus [XI ${ }^{e}-X I I^{e}$ siècles], Dax, 2004, $\mathrm{n}^{\circ} 142$ ). La traduction (reprise par ces éditeurs) et le commentaire qu'en a proposés Frédéric Boutoulle (« La paix et trêve de Dieu du Liber Rubeus », dans L'Église et la société dans le diocèse de Dax aux XI et XII siècles, éd. J. Cabanot et J.-B. Marquette, Dax, 2004, p. 47-72.) ne paraissent cependant pas entièrement satisfaisants.

Le lien qu'il suppose entre ces statuts et un concile de 1148 ou 1149 tenu à Mimizan demeure une hypothèse peu étayée. Ce concile est bien évoqué par une lettre de l'archevêque de Bordeaux, Geoffroi du Loroux, au régent Suger (RHF XV, p. 515), et il est vrai que celle-ci évoque une réunion de puissants enclins à la paix et au respect de la propriété ecclésiastique et royale, contre les atteintes du vicomte de Gabarret. Mais la requête (inquisitio) est accomplie sur ordre exprès du pape et du roi, ce que la charte des statuts du Liber Rubeus ne mentionne aucunement. La volonté de paix est un élément de langage très utilisé dans la communication politique et non une allusion assurée à la paix diocésaine et à la trêve de Dieu (la même réserve s'impose lorsque le vicomte de Dax, victime d'un meurtre, est dit voir été princeps pacis : F. Boutoulle, p. 65 de son commentaire). Enfin, F. Boutoulle traduit p. 66, dans cette lettre de Geoffroi du Loroux, statum terre nostre vobis siquidem ex condicto significare habuimus par « un statut de la terre qui y fut édicté par convention », ce qui lui semble une allusion directe aux statuts recopiés à Dax; cependant cela me semble vouloir dire seulement que « nous avions à vous informer comme convenu (ex condicto) de l'état de la terre », avant une excuse pour avoir différé de le faire. La date des statuts retrouvés au cartulaire de Dax demeure donc à placer, sans plus de précision, entre 1139 (concile de Latran II d'où procèdent les sauvegardes fondamentales de marchands, paysans et pèlerins et que suivent les mesures des années 1140 dans 
la province de Narbonne, qui ont pu se propager alors en Gascogne) et $1168 / 1169$ (mandement d'Alexandre III évoquant la paix et trêve de Dieu en Gascogne : Liber Rubeus, $\mathrm{n}^{\circ} 148$ ).

La traduction de statuts ou serments de paix est un exercice toujours difficile et celle de Frédéric Boutoulle pour ceux-ci n'est pas la seule à comporter quelques erreurs. Mais comme elle est canonisée par son adjonction à l'édition récente du cartulaire, il convient de les signaler. Pour Statutum est etiam ne pro dominis suis rustici capiantur ab aliquibus vel depredentur il comprend « que les paysans ne seraient ni pris ni mis au pillage par d'autres personnes que leur seigneur »; or il y a en latin pro dominis suis, et cette clause est pratiquement de fondation dans l'histoire des paix diocésaines : c'est l'interdiction de la vengeance indirecte entre seigneurs sur le dos des paysans. Un peu plus loin, il pense que les hospitaliers et templiers bénissent les bœufs, alors que signare doit signifier seulement marquer. Enfin, lorsque si treugua alicubi fracta fuerit, et violatores per inquisitionem episcopi vel fratrum supradictorum restaurare noluerint, il rend inquisitio par enquête, alors qu'il s'agit d'une requête (comme le montrent quatre textes gascons et toulousains de la même époque) et il convient donc de comprendre que « si la trêve est brisée en quelque endroit, et si ses violateurs se refusent à restituer les dommages à la requête de l'évêque ou des frères susdits $[\ldots] »$.

Ces statuts sont proches de ceux du Toulousain de 1163, retrouvés en 2018 par Nicolas Ruffini-Ronzani dans le fonds Giry de l'EPHE, même s'il y a de part et d'autre de vraies et intéressantes spécificités. À eux deux, ces textes récemment réapparus documentent une étape de la paix diocésaine, mal connue avant notre $\mathrm{XXI}^{\mathrm{e}}$ siècle, et dont l'identification même était rendue difficile par une méprise sur la date et l'attribution d'un mandement d'archevêque d'Auch, célèbre pour associer à la trêve de Dieu un appel à la mobilisation en guerre sainte contre les mercenaires. Or il m'apparaît que son attribution et sa datation doivent être rectifiées. Cet acte se trouvait au cartulaire de l'évêché de Lescar, aujourd'hui perdu, mais où Pierre de Marca a pu le copier ou le publier dans l'Histoire de Béarn, Paris, 1640, col. 887, réimpr. Pau, 1912, t. II, p. 80-81. Pierre de Marca pensait que son auteur était Guillaume I de Montaut (1068-1096), mais on a généralement, depuis lors, proposé plutôt Guillaume II d'Andozille (1126-1170) et les années suivant le concile de Latran II de 1139. Cette correction va certainement dans le bon sens, mais la datation du texte, ainsi modifiée, surprend encore puisqu'il lance un appel à la guerre sainte contre les mercenaires routiers, qui sont un fléau des années 1170 , dans les termes exacts du III ${ }^{\mathrm{e}}$ concile de Latran de 1179, en son canon 27 (distinct du canon 22 sur les sauvegardes et sur la trêve de Dieu). Malheureusement, en 1179 l'archevêque d'Auch ne s'appelait point Guillaume. Il est toutefois étonnant de voir que la trêve de Dieu est dans le texte celle de Latran III (Mansi XXII col. 229 : de la Septuagésime à l'octave de Pâques) et non de Latran II (Mansi XXI col. 529: de la Quinquagésime à l'octave de Pâques). Le directeur d'études examine de près l'Histoire de Béarn de Pierre de Marca, et observe que sa transcription, pour le premier mot, porte seulement l'initiale : $G$. Marca a compris qu'il s'agissait d'un Guillelmus, mais est-ce bien cela qu'il fallait lire? En fait, dans presque tous les cas, en Gascogne et ailleurs, au XII ${ }^{\mathrm{e}}$ siècle, Guillaume s'écrit Willelmus (ainsi pour Guillaume d'Andozille lui-même, au cartulaire de Dax, $\mathrm{n}^{\text {os }} 143$ 
et 146) et s'abrège dès lors en $W$. Rien ne prouve donc que $G$. soit un Guillaume, et il est même hautement probable que ce soit Geraldus, nom de l'archevêque de 1179, Géraud de Labarthe, lui aussi légat du pape et membre du groupe activement hostile aux hérétiques et aux mercenaires, emmené par Henri de Marcy, qui a inspiré le canon 27 de Latran III.

La paix diocésaine a eu en Gascogne une vie plus tardive et plus dense que prévu. Le mandement de l'archevêque Géraud de Labarthe, peu après 1179, comportant trêve de Dieu effective (avec des dates précises) et guerre sainte contre les mercenaires pestilentiels n'a pas eu d'équivalent connu de nous au Toulousain et en Albigeois, où pourtant une petite " croisade de Lavaur » s'est organisée contre des hérétiques. Et il est intéressant de relever qu'en 1227, le pape Grégoire IX enjoint à l'archevêque Amanieu d'Auch de relancer « la paix », ce qui semble bien signifier aussi la guerre sainte contre ses ennemis, c'est-à-dire contre Raymond VII et Toulouse, que viennent assiéger des « paroisse croisées » de Gascogne, comme nous l'apprend Guillaume de Puylaurens. Leur campagne pillarde correspond d'ailleurs au mode d'action le mieux attesté des communes diocésaines de ce temps : elles ont «talé » les terres des seigneurs ennemis de la paix, dans le diocèse de Mende au Gévaudan (dont la seconde conférence étudie attentivement tout le dossier, et spécialement l'enquête menée entre 1269 et 1278 et utilisée dans un mémoire élaboré pour le procès du paréage en 1301 / 1302).

La relance de ces armées d'évêques, pour quelques années, dans le sillage de la croisade albigeoise s'observe dans plusieurs diocèses méridionaux à partir de 1215 (II ${ }^{\mathrm{e}}$ concile de Montpellier, dont la seconde conférence regarde les canons), et elle pourrait bien expliquer pourquoi, entre 1226 et 1229, les croisés ont réussi à maintenir leurs positions jusqu'au succès final. 